\title{
Some new identities of Bernoulli, Euler and Hermite polynomials arising from umbral calculus
}

Dae San Kim', Taekyun Kim², Dmitry V Dolgy ${ }^{3}$ and Seog-Hoon Rim ${ }^{4}$

\section{"Correspondence:}

taekyun64@hotmail.com

2Department of Mathematics,

Kwangwoon University, Seoul,

139-701, Republic of Korea

Full list of author information is

available at the end of the article

\begin{abstract}
In this paper, we derive the identities of higher-order Bernoulli, Euler and Frobenius-Euler polynomials from the orthogonality of Hermite polynomials. Finally, we give some interesting and new identities of several special polynomials arising from umbral calculus.

MSC: 05A10;05A19
\end{abstract}

Keywords: Bernoulli polynomial; Euler polynomial; Abel polynomial

\section{Introduction}

The Hermite polynomials are defined by the generating function to be

$$
e^{2 x t-t^{2}}=e^{H(x) t}=\sum_{n=0}^{\infty} H_{n}(x) \frac{t^{n}}{n !}
$$

with the usual convention about replacing $H^{n}(x)$ by $H_{n}(x)$ (see [1]). In the special case, $x=0, H_{n}(0)=H_{n}$ are called the $n$th Hermite numbers. From (1.1) we have

$$
H_{n}(x)=(H+2 x)^{n}=\sum_{l=0}^{n}\left(\begin{array}{l}
n \\
l
\end{array}\right) H_{n-l} x^{l} 2^{l} .
$$

Thus, by (1.2), we get

$$
\frac{d^{k}}{d x^{k}} H_{n}(x)=2^{k}(n)_{k} H_{n-k}(x)=2^{k} \frac{n !}{(n-k) !} H_{n-k}(x),
$$

where $(x)_{k}=x(x-1) \cdots(x-k+1)$.

As is well known, the Bernoulli polynomials of order $r$ are defined by the generating function to be

$$
\left(\frac{t}{e^{t}-1}\right)^{r} e^{x t}=\sum_{n=0}^{\infty} B_{n}^{(r)}(x) \frac{t^{n}}{n !} \quad(r \in \mathbb{R}) .
$$

In the special case, $x=0, B_{n}^{(r)}(0)=B_{n}^{(r)}$ are called the $n$th Bernoulli numbers of order $r$ (see $[1-4])$.

(O) 2013 Kim et al.; licensee Springer. This is an Open Access article distributed under the terms of the Creative Commons Attribution License (http://creativecommons.org/licenses/by/2.0), which permits unrestricted use, distribution, and reproduction in any medium, provided the original work is properly cited. 
The Euler polynomials of order $r$ are also defined by the generating function to be

$$
\left(\frac{2}{e^{t}+1}\right)^{r} e^{x t}=\sum_{n=0}^{\infty} E_{n}^{(r)}(x) \frac{t^{n}}{n !} \quad(r \in \mathbb{R}) .
$$

In the special case, $x=0, E_{n}^{(r)}(0)=E_{n}^{(r)}$ are called the $n$th Euler numbers of order $r$.

For $\lambda(\neq 1) \in \mathbb{C}$, the Frobenius-Euler polynomials of order $r$ are given by

$$
\left(\frac{1-\lambda}{e^{t}-\lambda}\right)^{r} e^{x t}=\sum_{n=0}^{\infty} H_{n}^{(r)}(x \mid \lambda) \frac{t^{n}}{n !} \quad(r \in \mathbb{R}) .
$$

In the special case, $x=0, H_{n}^{(r)}(0 \mid \lambda)=H_{n}^{(r)}(\lambda)$ are called the $n$th Frobenius-Euler numbers of order $r$ (see [1-16]).

The Stirling numbers of the first kind are defined by the generating function to be

$$
(x)_{n}=\sum_{k=0}^{n} S_{1}(n, k) x^{k} \quad(\text { see }[11,14])
$$

and the Stirling numbers of the second kind are given by

$$
\left(e^{t}-1\right)^{n}=n ! \sum_{l=n}^{\infty} S_{2}(l, n) \frac{t^{l}}{l !} \quad(\text { see }[14])
$$

In [1] it is known that $H_{0}(x), H_{1}(x), \ldots, H_{n}(x)$ from an orthogonal basis for the space

$$
\mathbb{P}_{n}=\{p(x) \in \mathbb{Q}[x] \mid \operatorname{deg} p(x) \leq n\}
$$

with respect to the inner product

$$
\left\langle p_{1}(x), p_{2}(x)\right\rangle=\int_{-\infty}^{\infty} e^{-x^{2}} p_{1}(x) p_{2}(x) d x \quad(\text { see }[1])
$$

For $p(x) \in \mathbb{P}_{n}$, let us assume that

$$
p(x)=\sum_{k=0}^{n} C_{k} H_{k}(x)
$$

Then, from the orthogonality of Hermite polynomials and Rodrigues' formula, we have

$$
\begin{aligned}
C_{k} & =\frac{1}{2^{k} k ! \sqrt{\pi}} \int_{-\infty}^{\infty} e^{-x^{2}} H_{k}(x) p(x) d x \\
& =\frac{(-1)^{k}}{2^{k} k ! \sqrt{\pi}} \int_{-\infty}^{\infty}\left(\frac{d^{k}}{d x^{k}} e^{-x^{2}}\right) p(x) d x \quad \text { (see [1]). }
\end{aligned}
$$


In particular, for $p(x)=x^{m}(m \geq 0)$, we easily get

$$
\begin{aligned}
& \int_{-\infty}^{\infty}\left(\frac{d^{n}}{d x^{n}} e^{-x^{2}}\right) x^{m} d x \\
& \quad= \begin{cases}0 & \text { if } n>m \text { or } n \leq m \text { with } m-n \neq 0(\bmod 2), \\
\frac{(-1)^{n} m ! \sqrt{\pi}}{2^{m-n}\left(\frac{m-n}{2}\right) !} & \text { if } n \leq m \text { with } m-n \equiv 0(\bmod 2) .\end{cases}
\end{aligned}
$$

Let $\mathcal{F}$ be the set of all formal power series in the variable $t$ over $\mathbb{C}$ with

$$
\mathcal{F}=\left\{f(t)=\sum_{k=0}^{\infty} \frac{a_{k}}{k !} t^{k} \mid a_{k} \in \mathbb{C}\right\} \text {. }
$$

Let us assume that $\mathbb{P}$ is the algebra of polynomials in the variable $x$ over $\mathbb{C}$ and that $\mathbb{P}^{*}$ is the vector space of all linear functionals on $\mathbb{P} .\langle L \mid p(x)\rangle$ denotes the action of the linear functional $L$ on polynomials $p(x)$, and we remind that the vector space structure on $\mathbb{P}^{n}$ is defined by

$$
\begin{aligned}
& \langle L+M \mid p(x)\rangle=\langle L \mid p(x)\rangle+\langle M \mid p(x)\rangle, \\
& \langle c L \mid p(x)\rangle=c|L| p(x)\rangle,
\end{aligned}
$$

where $c$ is a complex constant (see $[2,11,14]$ ).

The formal power series

$$
f(t)=\sum_{k=0}^{\infty} \frac{a_{k}}{k !} t^{k} \in \mathcal{F}
$$

defines a linear functional on $\mathbb{P}$ by setting

$$
\left\langle f(t) \mid x^{n}\right\rangle=a_{n} \quad \text { for all } n \in \mathbb{Z}_{+}=\mathbb{N} \cup\{0\} .
$$

Thus, by (1.15) and (1.16), we get

$$
\left\langle t^{k} \mid x^{n}\right\rangle=n ! \delta_{n, k} \quad(n, k \geq 0)
$$

where $\delta_{n, k}$ is the Kronecker symbol (see $[2,11,14]$ ).

Let $f_{L}(t)=\sum_{k=0}^{\infty} \frac{\left\langle L \mid k^{k}\right\rangle}{k !} t^{k}$. By (1.16), we get

$$
\left\langle f_{L}(t) \mid x^{n}\right\rangle=\left\langle L \mid x^{n}\right\rangle, \quad n \geq 0 .
$$

Thus, by (1.18), we see that $f_{L}(t)=L$. The map $L \mapsto f_{L}(t)$ is a vector space isomorphism from $\mathbb{P}^{*}$ onto $\mathcal{F}$. Henceforth, $\mathcal{F}$ will be thought of as both a formal power series and a linear functional. We call $\mathcal{F}$ the umbral algebra. The umbral calculus is the study of umbral algebra (see $[2,11,14])$.

The order $o(f(t))$ of the nonzero power series $f(t)$ is the smallest integer $k$ for which the coefficient of $t^{k}$ does not vanish. A series $f(t)$ having $o(f(t))=1$ is called a delta series, and 
a series $f(t)$ having $o(f(t))=0$ is called an invertible series (see [2, 11, 14]). By (1.16) and (1.17), we see that $\left\langle e^{y t} \mid p(x)\right\rangle=p(y)$. For $f(t) \in \mathcal{F}$ and $p(x) \in \mathbb{P}$, we have

$$
f(t)=\sum_{k=0}^{\infty} \frac{\left\langle f(t) \mid x^{k}\right\rangle}{k !} t^{k}, \quad p(x)=\sum_{k=0}^{\infty} \frac{\left\langle t^{k} \mid p(x)\right\rangle}{k !} x^{k} .
$$

Let $f(t), g(t) \in \mathcal{F}$ and $p(x) \in \mathbb{P}$. Then we easily see that

$$
\langle f(t) g(t) \mid p(x)\rangle=\langle f(t) \mid g(t) p(x)\rangle=\langle g(t) \mid f(t) p(x)\rangle .
$$

From (1.19), we can derive the following equation:

$$
p^{(k)}(0)=\left\langle t^{k} \mid p(x)\right\rangle \quad \text { and } \quad\left\langle 1 \mid p^{(k)}(x)\right\rangle=p^{(k)}(0)
$$

Thus, by (1.21), we get

$$
t^{k} p(x)=p^{(k)}(x)=\frac{d^{k} p(x)}{d x^{k}} \quad(\text { see }[2,11,14]) .
$$

Let $f(t)$ be a delta series, and let $g(t)$ be an invertible series. Then there exists a unique sequence $S_{n}(x)$ of polynomials with $\left\langle g(t) f(t)^{k} \mid S_{n}(x)\right\rangle=n ! \delta_{n, k}$, where $n, k \geq 0$ (see $[2,11,14]$ ). The sequence $S_{n}(x)$ is called Sheffer sequence for $(g(t), f(t))$, which is denoted by $S_{n}(x) \sim$ $(g(t), f(t))$. For $f(t) \in \mathcal{F}$ and $p(x) \in \mathbb{P}$, we have

$$
\left\langle\frac{e^{y t}-1}{t} \mid p(x)\right\rangle=\int_{0}^{y} p(u) d u, \quad\left\langle e^{y t}-1 \mid p(x)\right\rangle=p(y)-p(0),
$$

and

$$
\langle f(t) \mid x p(x)\rangle=\left\langle f^{\prime}(t) \mid p(x)\right\rangle .
$$

In this paper, we introduce the identities of several special polynomials which are derived from the orthogonality of Hermite polynomials. Finally, we give some new and interesting identities of the higher-order Bernoulli, Euler and Frobenius-Euler polynomials arising from umbral calculus.

\section{Some identities of several special polynomials}

From (1.5), we note that

$$
\left(\frac{2}{e^{t}+1}\right)^{r}=\left(1+\frac{e^{t}-1}{2}\right)^{-r}=\sum_{j=0}^{\infty}\left(\begin{array}{c}
-r \\
j
\end{array}\right)\left(\frac{e^{t}-1}{2}\right)^{j}
$$

By (2.1), we get

$$
\begin{aligned}
\left(\frac{2}{e^{t}+1}\right)^{r} e^{x t} & =\sum_{j=0}^{\infty}\left(\begin{array}{c}
-r \\
j
\end{array}\right)\left(\frac{e^{t}-1}{2}\right)^{j} e^{x t} \\
& =\sum_{n=0}^{\infty}\left(\sum_{j=0}^{n}\left(\begin{array}{c}
-r \\
j
\end{array}\right)\left(\frac{e^{t}-1}{2}\right)^{j} x^{n}\right) \frac{t^{n}}{n !} .
\end{aligned}
$$


From (1.5) and (2.2), we have

$$
E_{n}^{(r)}(x)=\sum_{j=0}^{n}\left(\begin{array}{c}
-r \\
j
\end{array}\right) 2^{-j}\left(e^{t}-1\right)^{j} x^{n} .
$$

By (1.8) and (1.9), we get

$$
\begin{aligned}
\left(e^{t}-1\right)^{j} x^{n} & =\sum_{k=0}^{n-j} \frac{\left\langle t^{k} \mid\left(e^{t}-1\right)^{j} x^{n}\right\rangle}{k !}=\sum_{k=0}^{n-j} \frac{\left\langle\left(e^{t}-1\right)^{j} \mid t^{k} x^{n}\right\rangle}{k !} x^{k} \\
& =j ! \sum_{k=0}^{n-j}\left(\begin{array}{l}
n \\
k
\end{array}\right) \frac{\left\langle\left(e^{t}-1\right)^{j} \mid x^{n-k}\right\rangle}{j !} x^{k}=j ! \sum_{k=0}^{n-j}\left(\begin{array}{l}
n \\
j
\end{array}\right) S_{2}(n-k, j) x^{k} \\
& =j ! \sum_{k=j}^{n}\left(\begin{array}{l}
n \\
k
\end{array}\right) S_{2}(k, j) x^{n-k} .
\end{aligned}
$$

Therefore, by (2.3) and (2.4), we obtain the following theorem.

Theorem 2.1 For $n \geq 0$, we have

$$
\begin{aligned}
E_{n}^{(r)}(x) & =\sum_{0 \leq j \leq n} \sum_{j \leq k \leq n}\left(\begin{array}{l}
n \\
k
\end{array}\right)\left(\begin{array}{c}
-r \\
j
\end{array}\right) \frac{j !}{2^{j}} S_{2}(k, j) x^{n-k} \\
& =\sum_{0 \leq k \leq n}\left(\begin{array}{l}
n \\
k
\end{array}\right)\left[\sum_{0 \leq j \leq k}\left(\begin{array}{c}
-r \\
j
\end{array}\right) \frac{j !}{2^{j}} S_{2}(k, j)\right] x^{n-k} .
\end{aligned}
$$

By (1.5), we easily see that

$$
E_{n}^{(r)}(x)=\sum_{k=0}^{n}\left(\begin{array}{l}
n \\
k
\end{array}\right) E_{k}^{(r)} x^{n-k}
$$

Therefore, by Theorem 2.1 and (2.5), we obtain the following corollary.

Corollary 2.2 For $k \geq 0$, we have

$$
E_{k}^{(r)}=\sum_{j=0}^{k}\left(\begin{array}{c}
-r \\
j
\end{array}\right) \frac{j !}{2^{j}} S_{2}(k, j) .
$$

Let us take $p(x)=E_{n}^{(r)}(x) \in \mathbb{P}_{n}$. Then, by (1.11), we get

$$
E_{n}^{(r)}(x)=\sum_{k=0}^{n} C_{k} H_{k}(x)
$$

From (1.12), we can derive the computation of $C_{k}$ as follows:

$$
C_{k}=\frac{(-1)^{k}}{2^{k} k ! \sqrt{\pi}} \int_{-\infty}^{\infty}\left(\frac{d^{k} e^{-x^{2}}}{d x^{k}}\right) E_{n}^{(r)}(x) d x,
$$


where

$$
\begin{aligned}
& \int_{-\infty}^{\infty}\left(\frac{d^{k} e^{-x^{2}}}{d x^{k}}\right) E_{n}^{(r)}(x) d x \\
& =(-n)(-(n-1)) \cdots(-(n-k+1)) \int_{-\infty}^{\infty} e^{-x^{2}} E_{n-k}^{(r)}(x) d x \\
& =\frac{(-1)^{k} n !}{(n-k) !} \int_{-\infty}^{\infty} e^{-x^{2}} \sum_{l=0}^{n-k}\left(\begin{array}{c}
n-k \\
l
\end{array}\right) E_{n-k-l}^{(r)} x^{l} d x \\
& =\frac{(-1)^{k} n !}{(n-k) !} \sum_{l=0}^{n-k}\left(\begin{array}{c}
n-k \\
l
\end{array}\right) E_{n-k-l}^{(r)} \int_{-\infty}^{\infty} e^{-x^{2}} x^{l} d x \\
& =(-1)^{k} n ! \sqrt{\pi} \sum_{0 \leq l \leq n-k, l \text { :even }} \frac{1}{(n-k-l) ! 2^{l}\left(\frac{l}{2}\right) !} \sum_{j=0}^{n-k-l}\left(\begin{array}{c}
-r \\
j
\end{array}\right) \frac{j !}{2 j} S_{2}(n-k-l, j) .
\end{aligned}
$$

From (2.7) and (2.8), we can derive the following equation:

$$
\begin{aligned}
C_{k} & =n ! \sum_{0 \leq l \leq n-k, l \text { :even }} \frac{E_{n-k-l}^{(r)}}{k !(n-k-l) ! 2^{k+l}\left(\frac{l}{2}\right) !} \\
& =n ! \sum_{0 \leq l \leq n-k, l \text { :even }} \sum_{j=0}^{n-k-l} \frac{\left(\begin{array}{c}
-r \\
j
\end{array}\right) j ! S_{2}(n-k-l, j)}{k !(n-k-l) ! 2^{k+l+j}\left(\frac{l}{2}\right) !} .
\end{aligned}
$$

Therefore, by Corollary 2.2, (2.6) and (2.9), we obtain the following theorem.

Theorem 2.3 For $n \geq 0$, we have

$$
\begin{aligned}
E_{n}^{(r)}(x) & =n ! \sum_{k=0}^{n}\left\{\sum_{0 \leq l \leq n-k, l \text { :even }} \frac{E_{n-k-l}^{(r)}}{k !(n-k-l) ! 2^{k+l}\left(\frac{l}{2}\right) !}\right\} H_{k}(x) \\
& =n ! \sum_{k=0}^{n}\left\{\sum_{0 \leq l \leq n-k, l \text { :even }} \sum_{j=0}^{n-k-l} \frac{\left(\begin{array}{c}
-r \\
j
\end{array}\right) j ! S_{2}(n-k-l, j)}{k !(n-k-l) ! 2^{k+l+j}\left(\frac{l}{2}\right) !}\right\} H_{k}(x) .
\end{aligned}
$$

By (1.4), we easily see that

$$
\left(\frac{t}{e^{t}-1}\right)^{r}=\left(1+\frac{e^{t}-t-1}{t}\right)^{-r}=\sum_{j=0}^{\infty}\left(\begin{array}{c}
-r \\
j
\end{array}\right)\left(\frac{e^{t}-t-1}{t}\right)^{j} .
$$

Thus, by (2.10), we get

$$
\left(\frac{t}{e^{t}-1}\right)^{r} e^{x t}=\sum_{n=0}^{\infty}\left(\sum_{j=0}^{n}\left(\begin{array}{c}
-r \\
j
\end{array}\right)\left(\frac{e^{t}-t-1}{t}\right)^{j} x^{n}\right) \frac{t^{n}}{n !} .
$$

From (1.4) and (2.11), we have

$$
B_{n}^{(r)}(x)=\sum_{j=0}^{n}\left(\begin{array}{c}
-r \\
j
\end{array}\right)\left(\frac{e^{t}-t-1}{t}\right)^{j} x^{n} .
$$


By (1.19), we easily get

$$
\begin{aligned}
\left(\frac{e^{t}-t-1}{t}\right)^{j} x^{n} & =\sum_{k=0}^{n-j} \frac{\left\langle t^{k} \mid\left(\frac{e^{t}-t-1}{t}\right)^{j} x^{n}\right\rangle}{k !} x^{k} \\
& =\sum_{k=0}^{n-j} \frac{\left\langle\left(\frac{e^{t}-t-1}{t}\right)^{j} \mid t^{k} x^{n}\right\rangle}{k !} x^{k}=\sum_{k=0}^{n-j}\left(\begin{array}{l}
n \\
k
\end{array}\right) \sum_{l=0}^{j}\left(\begin{array}{l}
j \\
l
\end{array}\right)(-1)^{j-l}\left\langle\left(\frac{e^{t}-1}{t}\right)^{l} \mid x^{n-k}\right\rangle x^{k} \\
& \left.=\sum_{k=0}^{n-j}\left(\begin{array}{l}
n \\
k
\end{array}\right) \sum_{l=0}^{j}\left(\begin{array}{l}
j \\
l
\end{array}\right)(-1)^{j-l}\left\langle t^{0}\right|\left(\frac{e^{t}-1}{t}\right)^{l} x^{n-k}\right) x^{k} .
\end{aligned}
$$

From (1.8), (1.21) and (2.13), we have

$$
\left(\frac{e^{t}-t-1}{t}\right)^{j} x^{n}=\sum_{k=0}^{n-j} \sum_{l=0}^{j}\left(\begin{array}{l}
n \\
k
\end{array}\right)\left(\begin{array}{l}
j \\
l
\end{array}\right)(-1)^{j-l} \frac{(n-k) ! l !}{(n-k+l) !} S_{2}(n-k+l, l) x^{k}
$$

Thus, by (2.12) and (2.14), we get

$$
\begin{aligned}
B_{n}^{(r)}(x) & =\sum_{j=0}^{n} \sum_{k=0}^{n-j} \sum_{l=0}^{j}\left(\begin{array}{c}
-r \\
j
\end{array}\right)\left(\begin{array}{l}
n \\
k
\end{array}\right)\left(\begin{array}{l}
j \\
l
\end{array}\right)(-1)^{j-l} \frac{S_{2}(n-k+l, l)}{\left(\begin{array}{c}
n-k+l \\
l
\end{array}\right)} x^{k} \\
& =\sum_{k=0}^{n}\left(\begin{array}{l}
n \\
k
\end{array}\right)\left[\sum_{j=0}^{k} \sum_{l=0}^{j}\left(\begin{array}{c}
-r \\
j
\end{array}\right)\left(\begin{array}{l}
j \\
l
\end{array}\right) \frac{S_{2}(k+l, l)}{\left(\begin{array}{c}
k+l \\
l
\end{array}\right)}(-1)^{j-l}\right] x^{n-k} .
\end{aligned}
$$

Therefore, by (2.12) and (2.15), we obtain the following theorem.

Theorem 2.4 For $n \geq 0$, we have

$$
B_{n}^{(r)}(x)=\sum_{k=0}^{n}\left(\begin{array}{l}
n \\
k
\end{array}\right)\left[\sum_{j=0}^{k} \sum_{l=0}^{j}\left(\begin{array}{c}
-r \\
j
\end{array}\right)\left(\begin{array}{l}
j \\
l
\end{array}\right) \frac{S_{2}(k+l, l)}{\left(\begin{array}{c}
k+l \\
l
\end{array}\right)}(-1)^{j-l}\right] x^{n-k} .
$$

By (1.4), we easily get

$$
B_{n}^{(r)}(x)=\sum_{k=0}^{n}\left(\begin{array}{l}
n \\
k
\end{array}\right) B_{k}^{(r)} x^{n-k}
$$

Therefore, by Theorem 2.4 and (2.16), we obtain the following corollary.

Corollary 2.5 For $k \geq 0$, we have

$$
B_{k}^{(r)}=\sum_{j=0}^{k} \sum_{l=0}^{j}(-1)^{j-l}\left(\begin{array}{c}
-r \\
j
\end{array}\right)\left(\begin{array}{l}
j \\
l
\end{array}\right) \frac{S_{2}(k+l, l)}{\left(\begin{array}{c}
k+l \\
l
\end{array}\right)} .
$$

Let us consider $p(x)=B_{n}^{(r)}(x) \in \mathbb{P}_{n}$. Then, by $(1.11), B_{n}^{(r)}(x)$ can be written as

$$
B_{n}^{(r)}(x)=\sum_{k=0}^{n} C_{k} H_{k}(x)
$$


Now, we compute $C_{k}$ 's for $B_{k}^{(r)}(x)$ as follows:

$$
C_{k}=\frac{(-1)^{k}}{2^{k} k ! \sqrt{\pi}} \int_{-\infty}^{\infty}\left(\frac{d^{k} e^{-x^{2}}}{d x^{k}}\right) B_{n}^{(r)}(x) d x
$$

where

$$
\begin{aligned}
& \int_{-\infty}^{\infty}\left(\frac{d^{k} e^{-x^{2}}}{d x^{k}}\right) B_{n}^{(r)}(x) d x \\
& =(-n)(-(n-1)) \cdots(-(n-k+1)) \int_{-\infty}^{\infty} e^{-x^{2}} B_{n-k}^{(r)}(x) d x \\
& =\frac{(-1)^{k} n !}{(n-k) !} \sum_{l=0}^{n-k}\left(\begin{array}{c}
n-k \\
l
\end{array}\right) B_{n-k-l}^{(r)} \int_{-\infty}^{\infty} e^{-x^{2}} x^{l} d x \\
& =(-1)^{k} n ! \sqrt{\pi} \sum_{0 \leq l \leq n-k, l: \text { even }} \frac{B_{n-k-l}^{(r)}}{(n-k-l) ! 2^{l}\left(\frac{l}{2}\right) !} .
\end{aligned}
$$

By Corollary 2.5 and (2.19), we get

$$
\begin{aligned}
& \int_{-\infty}^{\infty}\left(\frac{d^{k} e^{-x^{2}}}{d x^{k}}\right) B_{n}^{(r)}(x) d x \\
& =(-1)^{k} n ! \sqrt{\pi} \sum_{0 \leq l \leq n-k, l \text { :even }} s \sum_{j=0}^{n-k-l} \sum_{m=0}^{j} \frac{(-1)^{j-m}\left(\begin{array}{c}
-r \\
j
\end{array}\right)\left(\begin{array}{c}
j \\
m
\end{array}\right) S_{2}(n-k-l+m, m)}{(n-k-l) ! 2^{l}\left(\frac{l}{2}\right) !\left(\begin{array}{c}
n-k-l+m \\
m
\end{array}\right)}
\end{aligned}
$$

From (2.18) and (2.20), we have

$$
\begin{aligned}
C_{k} & =n ! \sum_{0 \leq l \leq n-k, l: \text { even }} \frac{B_{n-k-l}^{(r)}}{(n-k-l) ! k ! 2^{k+l}\left(\frac{l}{2}\right) !} \\
& =n ! \sum_{0 \leq l \leq n-k, l \text { :even }} \sum_{j=0}^{n-k-l} \sum_{m=0}^{j} \frac{(-1)^{j-m}\left(\begin{array}{c}
-r \\
j
\end{array}\right)\left(\begin{array}{c}
j \\
m
\end{array}\right) S_{2}(n-k-l+m, m)}{(n-k-l) ! k ! 2^{k+l}\left(\frac{l}{2}\right) !\left(\begin{array}{c}
n-k-l+m \\
m
\end{array}\right)} .
\end{aligned}
$$

Therefore, by (2.17) and (2.21), we obtain the following theorem.

Theorem 2.6 For $n \geq 0$, we have

$$
\begin{aligned}
B_{n}^{(r)}(x) & =n ! \sum_{k=0}^{n}\left\{\sum_{0 \leq l \leq n-k, l \text { :even }} \frac{B_{n-k-l}^{(r)}}{(n-k-l) ! k ! 2^{k+l}\left(\frac{l}{2}\right) !}\right\} H_{k}(x) \\
& =n ! \sum_{k=0}^{n}\left\{\sum_{0 \leq l \leq n-k, l \text { :even }} \sum_{j=0}^{n-k-l} \sum_{m=0}^{j} \frac{(-1)^{j-m}\left(\begin{array}{c}
-r \\
j
\end{array}\right)\left(\begin{array}{c}
j \\
m
\end{array}\right) S_{2}(n-k-l+m, m)}{(n-k-l) ! k ! 2^{k+l}\left(\frac{l}{2}\right) !\left(\begin{array}{c}
n-k-l+m \\
m
\end{array}\right)}\right\} H_{k}(x) .
\end{aligned}
$$

It is easy to show that

$$
\left(\frac{1-\lambda}{e^{t}-\lambda}\right)^{r}=\left(1+\frac{e^{t}-1}{1-\lambda}\right)^{-r}=\sum_{j=0}^{\infty}\left(\begin{array}{c}
-r \\
j
\end{array}\right)\left(\frac{1}{1-\lambda}\right)^{j}\left(e^{t}-1\right)^{j} .
$$


From (1.6) and (2.22), we have

$$
H_{n}^{(r)}(x \mid \lambda)=\sum_{j=0}^{n}\left(\begin{array}{c}
-r \\
j
\end{array}\right)(1-\lambda)^{-j}\left(e^{t}-1\right)^{j} x^{n}
$$

where

$$
\begin{aligned}
\left(e^{t}-1\right)^{j} x^{n} & =j ! \sum_{k=j}^{\infty} S_{2}(k, j) \frac{t^{k}}{k !} x^{n} \\
& =j ! \sum_{k=j}^{n}\left(\begin{array}{l}
n \\
k
\end{array}\right) S_{2}(k, j) x^{n-k} .
\end{aligned}
$$

Thus, by (2.24), we get

$$
\left(e^{t}-1\right)^{j} x^{n}=j ! \sum_{k=j}^{n}\left(\begin{array}{l}
n \\
k
\end{array}\right) S_{2}(k, j) x^{n-k} .
$$

From (2.23) and (2.25), we can derive the following equation:

$$
\begin{aligned}
& H_{n}^{(r)}(x \mid \lambda)=\sum_{j=0}^{n} \sum_{k=j}^{n}\left(\begin{array}{l}
n \\
k
\end{array}\right)\left(\begin{array}{c}
-r \\
j
\end{array}\right) \frac{j !}{(1-\lambda)^{j}} S_{2}(k, j) x^{n-k} \\
& =\sum_{k=0}^{n} \sum_{j=0}^{k}\left(\begin{array}{l}
n \\
k
\end{array}\right)\left(\begin{array}{c}
-r \\
j
\end{array}\right) \frac{j !}{(1-\lambda)^{j}} S_{2}(k, j) x^{n-k} \\
& =\sum_{k=0}^{n}\left(\begin{array}{l}
n \\
k
\end{array}\right)\left[\sum_{j=0}^{k}\left(\begin{array}{c}
-r \\
j
\end{array}\right) \frac{j !}{(1-\lambda)^{j}} S_{2}(k, j)\right] x^{n-k} .
\end{aligned}
$$

By (1.6), we easily see that

$$
H_{n}^{(r)}(x \mid \lambda)=\sum_{k=0}^{n}\left(\begin{array}{l}
n \\
k
\end{array}\right) H_{k}^{(r)}(\lambda) x^{n-k} .
$$

Therefore, by (2.26) and (2.27), we obtain the following theorem.

Theorem 2.7 For $k \geq 0$, we have

$$
H_{k}^{(r)}(\lambda)=\sum_{j=0}^{k}\left(\begin{array}{c}
-r \\
j
\end{array}\right) \frac{j !}{(1-\lambda)^{j}} S_{2}(k, j) .
$$

Let us take $p(x)=H_{n}^{(r)}(x \mid \lambda) \in \mathbb{P}_{n}$. Then, by (1.11), $H_{n}^{(r)}(x \mid \lambda)$ is given by

$$
H_{n}^{(r)}(x \mid \lambda)=\sum_{k=0}^{n} C_{k} H_{k}(x) .
$$


By (1.12), we get

$$
C_{k}=\frac{(-1)^{k}}{2^{k} k ! \sqrt{\pi}} \int_{-\infty}^{\infty}\left(\frac{d^{k} e^{-x^{2}}}{d x^{k}}\right) H_{n}^{(r)}(x \mid \lambda) d x,
$$

where

$$
\begin{aligned}
& \int_{-\infty}^{\infty}\left(\frac{d^{k} e^{-x^{2}}}{d x^{k}}\right) H_{n}^{(r)}(x \mid \lambda) d x \\
& =\frac{(-1)^{k} n !}{(n-k) !} \sum_{l=0}^{n-k}\left(\begin{array}{c}
n-k \\
l
\end{array}\right) H_{n-k-l}^{(r)}(\lambda) \int_{-\infty}^{\infty} e^{-x^{2}} x^{l} d x \\
& =(-1)^{k} n ! \sqrt{\pi} \sum_{0 \leq l \leq n-k, l: \text { even }} \frac{H_{n-k-l}^{(r)}(\lambda)}{(n-k-l) ! 2^{l}\left(\frac{l}{2}\right) !} \\
& =(-1)^{k} n ! \sqrt{\pi} \sum_{0 \leq l \leq n-k, l: \text { even }} \sum_{j=0}^{n-k-l} \frac{\left(\begin{array}{c}
-r \\
j
\end{array}\right) j ! S_{2}(n-k-l, j)}{(n-k-l) ! 2^{l}(1-\lambda)^{j}\left(\frac{l}{2}\right) !} .
\end{aligned}
$$

By (2.29) and (2.30), we get

$$
\begin{aligned}
C_{k} & =n ! \sum_{0 \leq l \leq n-k, l: \text { even }} \frac{H_{n-k-l}^{(r)}(\lambda)}{(n-k-l) ! k ! 2^{l+k}\left(\frac{l}{2}\right) !} \\
& =n ! \sum_{0 \leq l \leq n-k, l \text { :even }} \sum_{j=0}^{n-k-l} \frac{\left(\begin{array}{c}
-r \\
j
\end{array}\right) j ! S_{2}(n-k-l, j)}{(n-k-l) ! k ! 2^{k+l}(1-\lambda) j\left(\frac{l}{2}\right) !} .
\end{aligned}
$$

Therefore, by (2.28) and (2.31), we obtain the following theorem.

Corollary 2.8 For $n \geq 0$, we have

$$
\begin{aligned}
H_{n}^{(r)}(x \mid \lambda) & =n ! \sum_{k=0}^{n}\left\{\sum_{0 \leq l \leq n-k, l: \text { even }} \frac{H_{n-k-l}^{(r)}(\lambda)}{(n-k-l) ! k ! 2^{l+k}\left(\frac{l}{2}\right) !}\right\} H_{k}(x) \\
& =n ! \sum_{k=0}^{n}\left\{\sum_{0 \leq l \leq n-k, l: \text { even }} \sum_{j=0}^{n-k-l} \frac{\left(\begin{array}{c}
-r \\
j
\end{array}\right) j ! S_{2}(n-k-l, j)}{(n-k-l) ! k ! 2^{k+l}(1-\lambda)^{j}\left(\frac{l}{2}\right) !}\right\} H_{k}(x) .
\end{aligned}
$$

\section{Competing interests}

The authors declare that they have no competing interests.

\section{Authors' contributions}

All authors contributed equally to the manuscript and typed, read, and approved the final manuscript.

\section{Author details}

'Department of Mathematics, Sogang University, Seoul, 121-742, Republic of Korea. ${ }^{2}$ Department of Mathematics, Kwangwoon University, Seoul, 139-701, Republic of Korea. ${ }^{3}$ Hanrimwon, Kwangwoon University, Seoul, 139-701, Republic of Korea. ${ }^{4}$ Department of Mathematics Education, Kyungpook National University, Taegu, 702-701, Republic of Korea.

\section{Acknowledgements}

The authors would like to express their sincere gratitude to referees for their valuable comments. This paper is supported in part by the Research Grant of Kwangwoon University in 2013. 


\section{References}

1. Kim, DS, Kim, T, Rim, SH, Lee, S-H: Hermite polynomials and their applications associated with Bernoulli and Euler numbers. Discrete Dyn. Nat. Soc. 2012, Article ID 974632, 13 pp. (2012). doi:10.1155/2012/974632

2. Kim, T: Symmetry $p$-adic invariant integral on $\mathbb{Z}_{p}$ for Bernoulli and Euler polynomials. J. Differ. Equ. Appl. 14 1267-1277 (2008)

3. Kim, T, Choi, J, Kim, YH, Ryoo, CS: On q-Bernstein and q-Hermite polynomials. Proc. Jangjeon Math. Soc. 14(2), $215-221$ (2011)

4. Ryoo, C: Some relations between twisted $q$-Euler numbers and Bernstein polynomials. Adv. Stud. Contemp. Math. 21(2), 217-223 (2011)

5. Araci, S, Acikgoz, M: A note on the Frobenius-Euler numbers and polynomials associated with Bernstein polynomials. Adv. Stud. Contemp. Math. 22(3), 399-406 (2012)

6. Araci, S, Erdal, D, Seo, J: A study on the fermionic $p$-adic $q$-integral representation on $\mathbb{Z}_{p}$ associated with weighted q-Bernstein and q-Genocchi polynomials. Abstr. Appl. Anal. 2011, Article ID 649248, 10 pp. (2011)

7. Bayad, A: Modular properties of elliptic Bernoulli and Euler functions. Adv. Stud. Contemp. Math. 20(3), 389-401 (2010)

8. Can, M, Cenkci, M, Kurt, V, Simsek, Y: Twisted Dedekind type sums associated with Barnes' type multiple Frobenius-Euler-functions. Adv. Stud. Contemp. Math. 18(2), 135-160 (2009)

9. Carlitz, L: The product of two Eulerian polynomials. Math. Mag. 368, 37-41 (1963)

10. Ding, D, Yang, J: Some identities related to the Apostol-Euler and Apostol-Bernoulli polynomials. Adv. Stud. Contemp. Math. 20(1), 7-21 (2010)

11. Kim, DS, Kim, T: Some identities of Frobenius-Euler polynomials arising from umbral calculus. Adv. Differ. Equ. 2012, 196 (2012). doi:10.1186/1687-1847-2012-196

12. Ozden, H, Cangul, IN, Simsek, Y: Remarks on q-Bernoulli numbers associated with Daehee numbers. Adv. Stud. Contemp. Math. 18(1), 41-48 (2009)

13. Rim, S-H, Joung, J, Jin, J-H, Lee, S-J: A note on the weighted Carlitz's type $q$-Euler numbers and $q$-Bernstein polynomials. Proc. Jangjeon Math. Soc. 15, 195-201 (2012)

14. Roman, S: The Umbral Calculus. Dover, New York (2005)

15. Kim, T: An identity of the symmetry for the Frobenius-Euler polynomials associated with the fermionic $p$-adic invariant $q$-integrals on $\mathbb{Z}_{p}$. Rocky Mt. J. Math. 41, 239-247 (2011)

16. Simsek, Y: Generating functions of the twisted Bernoulli numbers and polynomials associated with their interpolation functions. Adv. Stud. Contemp. Math. 16(2), 251-278 (2008)

doi:10.1186/1687-1847-2013-73

Cite this article as: Kim et al.: Some new identities of Bernoulli, Euler and Hermite polynomials arising from umbra calculus. Advances in Difference Equations 2013 2013:73.

\section{Submit your manuscript to a SpringerOpen ${ }^{\circ}$ journal and benefit from:}

- Convenient online submission

- Rigorous peer review

- Immediate publication on acceptance

- Open access: articles freely available online

- High visibility within the field

- Retaining the copyright to your article 